\title{
A high-sensitivity phospho-switch triggered by Cdk1 governs chromosome morphogenesis during cell division
}

\author{
Xavier Robellet, ${ }^{1,2,4}$ Yogitha Thattikota, ${ }^{1,2,4}$ Fang Wang, ${ }^{1,2}$ Tse-Luen Wee, ${ }^{3}$ Mirela Pascariu, ${ }^{1,2}$ \\ Sahana Shankar, ${ }^{1,2}$ Éric Bonneil, ${ }^{1}$ Claire M. Brown, ${ }^{3}$ and Damien D'Amours ${ }^{1,2}$ \\ ${ }^{1}$ Institute for Research in Immunology and Cancer (IRIC), ${ }^{2}$ Département de Pathologie et Biologie Cellulaire, Université de \\ Montréal, Montréal, Quebec H3C 3J7, Canada; ${ }^{3}$ Advanced BioImaging Facility (ABIF), Department of Physiology, McGill \\ University, Montréal, Quebec H3G 0B1, Canada
}

\begin{abstract}
The initiation of chromosome morphogenesis marks the beginning of mitosis in all eukaryotic cells. Although many effectors of chromatin compaction have been reported, the nature and design of the essential trigger for global chromosome assembly remain unknown. Here we reveal the identity of the core mechanism responsible for chromosome morphogenesis in early mitosis. We show that the unique sensitivity of the chromosome condensation machinery for the kinase activity of Cdk1 acts as a major driving force for the compaction of chromatin at mitotic entry. This sensitivity is imparted by multisite phosphorylation of a conserved chromatinbinding sensor, the Smc4 protein. The multisite phosphorylation of this sensor integrates the activation state of Cdk1 with the dynamic binding of the condensation machinery to chromatin. Abrogation of this event leads to chromosome segregation defects and lethality, while moderate reduction reveals the existence of a novel chromatin transition state specific to mitosis, the intertwist configuration. Collectively, our results identify the mechanistic basis governing chromosome morphogenesis in early mitosis and how distinct chromatin compaction states can be established via specific thresholds of Cdk1 kinase activity.
\end{abstract}

[Keywords: chromosome; morphogenesis; Cdk1; Smc4; multisite phosphorylation]

Supplemental material is available for this article.

Received September 27, 2014; revised version accepted January 14, 2015.

In his seminal description of mitosis, Flemming (1882) recognized that the formation of visible chromosomes is one of the earliest cytological landmarks of the cell division program. Since then, much effort has been devoted to unraveling the structural and regulatory mechanisms that underpin the formation of mitotic and meiotic chromosomes (for review, see Maeshima and Eltsov 2008; Baxter and Aragon 2012; Hirano 2012). A number of independent steps-such as chromosome replication, condensation, and the establishment of sister chromatid cohesion-are required for the formation of mature and functional chromosomes during cell division. The morphological changes that associate with these steps are collectively referred to as the process of chromosome morphogenesis (van Heemst et al. 1999; Yu and Koshland 2005).

The compaction of amorphous chromatin into visible chromosomes is one of the earliest and most extensive changes in the morphogenetic process (Flemming 1882).

${ }^{4}$ These authors contributed equally to this work. Corresponding author: damien.damours@umontreal.ca Article is online at http://www.genesdev.org/cgi/doi/10.1101/gad.253294.114.
Given the physical challenges associated with the assembly of micrometer-scale chromosomes in the crowded cellular environment (Marko 2008), it is not surprising that many chromatin and cell cycle effectors have been suggested as possible regulators of the process. Chief among those is the condensin complex, a pentameric ATPase that binds to chromatin and alters its configuration and/or association status with distant chromatin regions (Bazile et al. 2010; Baxter and Aragon 2012; Hirano 2012). Other factors, such as cell cycle kinases and histone-modifying enzymes, have also been proposed as possible regulators of chromosome condensation during mitosis (Morishita et al. 2001; St-Pierre et al. 2009; Abe et al. 2011; Neurohr et al. 2011; Wilkins et al. 2014). Although it is clear that these enzymes impact chromatin compaction at specific genomic locations and/or during specific stages of mitosis, it is remarkable that

(C) 2015 Robellet et al. This article is distributed exclusively by Cold Spring Harbor Laboratory Press for the first six months after the full-issue publication date (see http://genesdev.cshlp.org/site/misc/terms.xhtml). After six months, it is available under a Creative Commons License (Attribution-NonCommercial 4.0 International), as described at http:// creativecommons.org/licenses/by-nc/4.0/. 
the chromosome condensation process as a whole remains largely operational when these enzymes are fully inhibited in mammalian cells (Cimini et al. 2003; Ditchfield et al. 2003; Hauf et al. 2003; Ono et al. 2004; Lenart et al. 2007; Abe et al. 2011). The fact that no specific mutations and/or inhibitory conditions prevent the formation of condensed chromosomes during metaphase in mammalian cells suggests that the fundamental nature of the mechanism responsible for global chromosome assembly is still unknown.

Another key issue about chromosome morphogenesis relates to the timing of the process in relationship to other mitotic events. It was established several decades ago that mitosis is initiated by a sudden increase in Cdk1 activity (Morgan 2007). The fact that the early assembly of mitotic chromosomes correlates well with the early increase in Cdk1 activity in prophase /Gavet and Pines 2010) suggests that Cdk1 may regulate early chromosome assembly directly. However, the dependency of the chromosome morphogenesis process on mitotic entry (Vassilev et al. 2006; Paulson 2007; Gong and Ferrell 2010) makes it difficult to determine whether the impact of Cdk1 on chromosome morphology reflects a direct role in this process or, alternatively, a need to establish a mitotic state prior to initiating chromosome assembly. Moreover, given that a requirement for Cdk1 activity is shared between many mitotic processes, it is unclear why the establishment of chromosome condensation should precede other mitotic landmarks if all mitotic processes respond to the same Cdk1 signal. The temporal primacy of condensation in the mitotic program could be due to heightened sensitivity to Cdk1 phosphorylation, higher specificity for specific cyclinCdk1 complexes, or a yet-unknown Cdk1-independent mechanism. In this study, we tested those possibilities and showed how Cdk1 can initiate chromosome morphogenesis directly using quantitative multisite phosphorylation of the Smc4 protein. Moreover, we identified a novel two-step mechanism necessary for the folding of chromatin and subsequent assembly of functional chromosome during mitosis.

\section{Results}

\section{Regulation of chromosome morphology by Cdk1}

To investigate the mechanistic basis for chromosome morphogenesis, we first determined whether the process was under direct Cdk1 control in Saccharomyces cerevisiae. Yeast cells carrying a Cdk1 temperature-sensitive (ts) mutation, cdc28-4, were arrested in mitosis, and the morphology of the ribosomal DNA (rDNA) locus was evaluated in these cells. The shape of the rDNA locus is dramatically reorganized during mitosis, which provides a sensitive assay to monitor chromosome morphogenesis in yeast (Guacci et al. 1994). Whereas wild-type cells arrested in mid-mitosis showed the typical condensed "loop" configuration of the rDNA locus at both $23^{\circ} \mathrm{C}$ and $37^{\circ} \mathrm{C}$, inactivation of $\mathrm{Cdk} 1$ resulted in the formation of an uncondensed "puff" rDNA signal at $37^{\circ} \mathrm{C}$ (Fig. 1A; Guacci et al. 1994). Having established the Cdk1 dependency of the chromosome morphogenesis process in yeast, we next asked whether chromosome condensation could be quantitatively modulated by down-regulation of Cdk1 activity using conditional B-type cyclin mutations (i.e., clb1 clb3 clb4 clb2-VI; clb-ts mutant henceforth). Although able to enter mitosis, $c l b$-ts cells are incapable of executing subsequent mitotic events (Amon et al. 1993). Analysis of chromosome morphology in this mutant revealed the existence of a novel intertwined rDNA configuration distinct from the uncondensed "puff" signal (Fig. 1B) or the fully condensed loop signal (Fig. 1A). Specifically, under low Cdk1 activity, individual chromosomal "threads" are clearly visible at the rDNA locus and appear to follow an elaborate intertwined path distinct from the nonoverlapping path of chromosome threads in the loop configuration (Fig. 1A,B; Supplemental Fig. S1). We therefore refer to this novel stage in chromosome condensation as the intertwist configuration. Interestingly, chromatin folding within the intertwist configuration is consistent in shape with the early condensation intermediates that were recently proposed to exist based on polymer simulation models (Naumova et al. 2013). Cytological characterization of $c l b$-ts mutants confirmed that other mitotic events, such as bipolar spindle formation and chromosome segregation, do not occur in these cells (Supplemental Fig. S1; Amon et al. 1993).

To exclude the possibility that rDNA intertwist formation is a consequence of a change in Cdk1 specificity in $c l b$ ts mutants, we monitored chromosome morphology in the cdc28-as1 mutant. This mutant, when treated with low concentrations of 1NM-PP1 inhibitor, experiences a cell cycle arrest at mitotic entry (i.e., after DNA replication but prior to mitotic spindle formation) (Bishop et al. 2000) similar to the point of arrest of $c l b$-ts mutants. Examination of chromosome morphology in cdc28-as1 cells treated with the inhibitor revealed a striking enrichment in the number of cells carrying the intertwist configuration at the rDNA, whereas untreated cells formed mostly loops at this locus under identical conditions (Fig. 1C). Interestingly, the intertwist configuration appears to be stabilized at low temperature and could be readily observed in wild-type cells progressing synchronously into mitosis at $16^{\circ} \mathrm{C}$ (Supplemental Fig. S2). As previously observed with the fully condensed loop configuration (Lavoie et al. 2004), formation of the intertwist rDNA intermediate also requires cohesin activity, since inactivation of mcd1-1 prevented the appearance of this rDNA configuration in mitosis (Supplemental Fig. S3). Taken together, our results indicate that chromosome condensation is initiated at levels of Cdk1 activity that are too low to induce other mitotic events. Moreover, conditions of low Cdk1 activity revealed the existence of a hitherto unknown early chromatinfolding step in the formation of mitotic chromosomes.

\section{The Smc4 subunit of condensin is a target for Cdk1 in early mitosis}

What is the target of Cdk1 in the induction of chromosome morphogenesis? A likely candidate is the condensin 
Robellet et al.

A

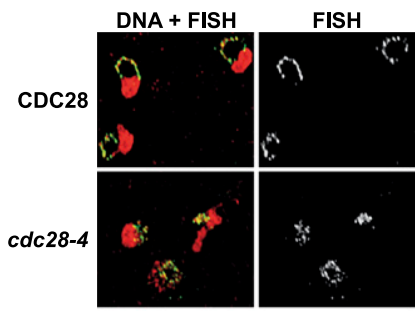

B

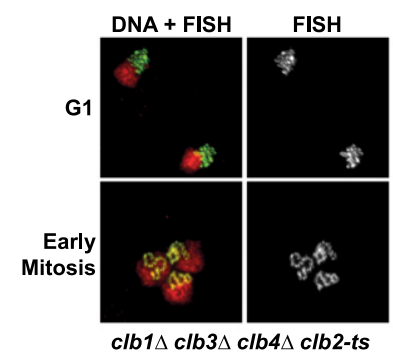

C

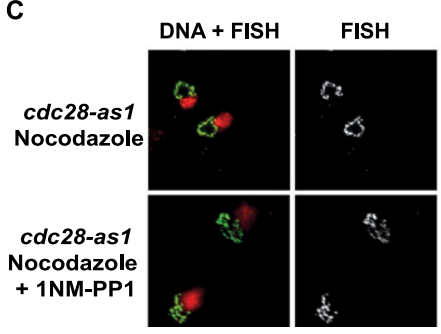

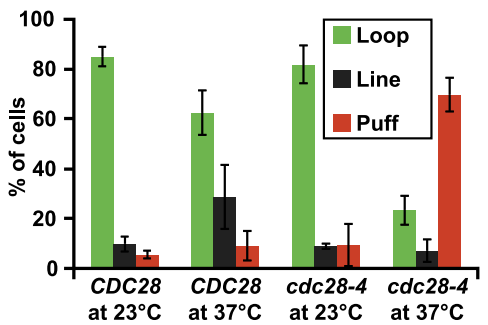
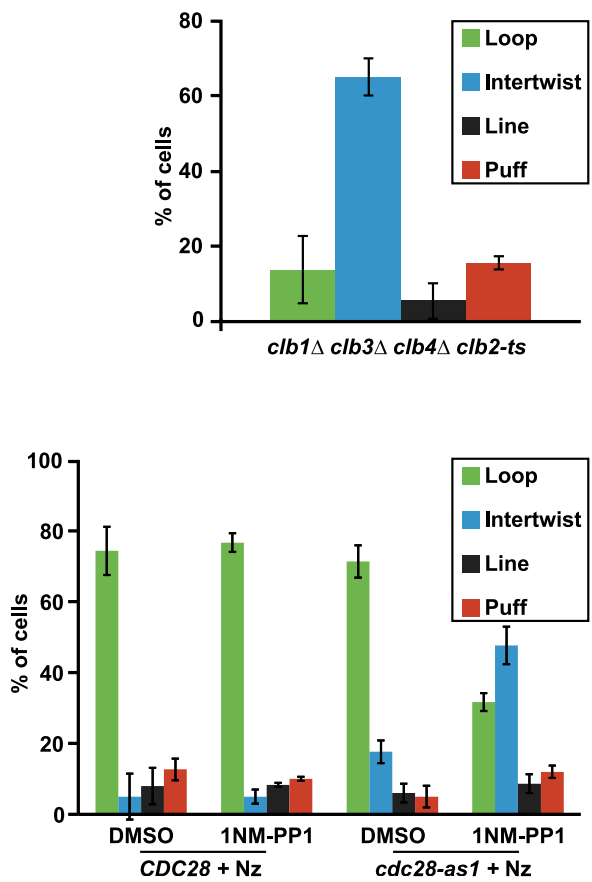

Figure 1. Modulation of Cdk1 activity unveils distinct steps in the process of chromosome morphogenesis. $(A-C)$ The morphology of the yeast rDNA locus was revealed by fluorescence in situ hybridization (FISH). Representative micrographs of the most prominent rDNA morphology observed for each condition are shown at the left. Propidium iodide (PI; red) and fluorescein isothiocyanate (FITC; green) were used to label the nucleus and rDNA locus, respectively. Quantification of each rDNA species is shown at the right. At least 100 nuclei were counted per condition ( $n=3$ for all experiments). Error bars represent SD. (A) cdc28-4 mutants exhibit classic condensation defects. Cells were blocked in metaphase at $23^{\circ} \mathrm{C}$ using nocodazole and shifted for $1 \mathrm{~h}$ to $37^{\circ} \mathrm{C}$ before processing of the samples for FISH analysis. To ensure that the quantification reflects loss of condensation in mitotic cells rather than a return to interphase due to the loss of cdc28-4 activity (Sanchez-Diaz et al. 2012), we normalized the rDNA quantification according to the budding index of cells during the arrest. $(B, C)$ Reducing Cdk1 activity uncovers a novel condensation intermediate at the rDNA. (B) clb-ts cells growing asynchronously at $23^{\circ} \mathrm{C}$ were arrested in G1 or early mitosis by incubation with $\alpha$ factor or by shifting the culture to $37^{\circ} \mathrm{C}$ for $135 \mathrm{~min}$, respectively. Cells were subsequently harvested, and their rDNA morphology was revealed by FISH. $(C)$ cdc28-as1 cells growing asynchronously at $25^{\circ} \mathrm{C}$ were arrested in early mitosis by incubation with nocodazole with or without the kinase inhibitor NM-PP1 for $150 \mathrm{~min}$. Samples were then fixed, and the morphology of the rDNA locus was monitored as above. complex, a central effector of chromosome condensation in eukaryotes (for review, see Baxter and Aragon 2012; Hirano 2012). To test this possibility, we removed all of the core Cdk1 consensus sites (i.e., Ser/Thr-Pro) (Holt et al. 2009) from condensin subunits and determined the effect of these mutations on cell proliferation (Fig. 2A,B). Only smc4-10A showed detectable growth defects in the absence of Cdk1 phosphorylation (Fig. 2B). Combining all mutations in one yeast strain had only modest additive effects on cell proliferation relative to the smc4-10A single mutant (Fig. 2B). These results indicate that the Smc4 subunit of condensin is a likely target of Cdk1 in vivo. To further substantiate this notion, we immunopurified the condensin complex from metaphase-arrested cells and subjected the immunoprecipitate to mass spectrometry analysis to identify possible in vivo phosphorylation sites. This analysis revealed the existence of five phosphorylation sites that conform to the Cdk1 consensus in Smc4 (Fig. 2C; Supplemental Fig. S4A) and none in the other subunits of condensin. An additional Cdk1 phospho-site, Ser117, was uncovered in Smc4 in proteome-wide analyses of mitotic cells (Holt et al. 2009; Kao et al. 2014). Interestingly, all of these Cdk1 phosphosites were clustered in the N-terminal extension of
Smc4, a region of the protein that is conserved among eukaryotic Smc4 family members but absent in the Smc1-3 families (Supplemental Fig. S4B,C). Deletion of the N-terminal extension of Smc4 results in a stable but inactive protein, thereby revealing the essential role played by this part of Smc4 in condensin function (Fig. 2D). Finally, we asked whether Cdk1 is directly responsible for condensin phosphorylation. To test this possibility, we purified condensin from yeast and exposed it to purified Cdk1-Clb2 in the presence of radiolabeled ATP. We observed that a single band corresponding to the molecular mass of Smc4 became phosphorylated following the kinase reaction (Fig. 2E; St-Pierre et al. 2009). Performing a similar experiment using only the N-terminal fragment of Smc4 (residues 1-163) resulted in a Cdk1 phosphorylation-induced gel retardation of the substrate after electrophoresis (Fig. 2F). Taken together, these experiments demonstrate that Cdk1 phosphorylates Smc4 in vitro and in vivo.

We next characterized the timing of Smc4 phosphorylation during the cell cycle. Since phosphorylation of the $\mathrm{N}$-terminal part of Smc4 by Cdk1 causes a gel shift after electrophoresis (Fig. 2F), we used cells expressing the $\mathrm{N}$-terminal extension of Smc4 fused to an epitope tag 
A

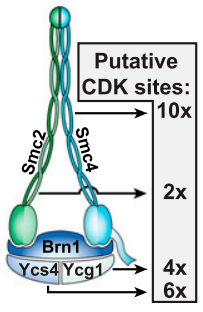

C

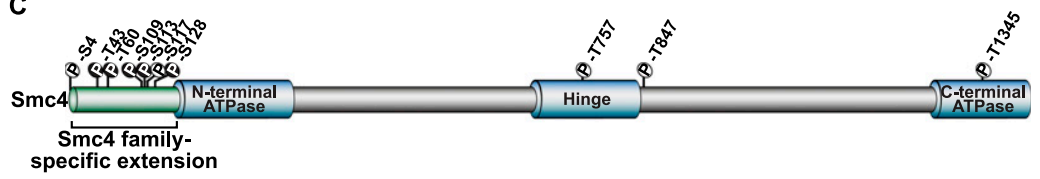

D

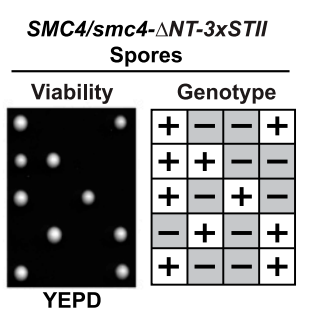

E

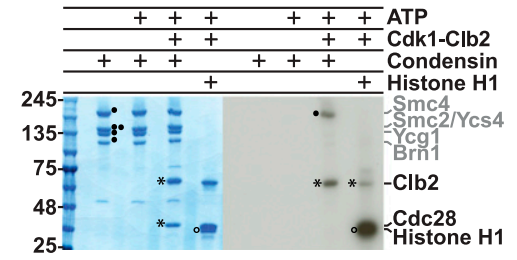

B
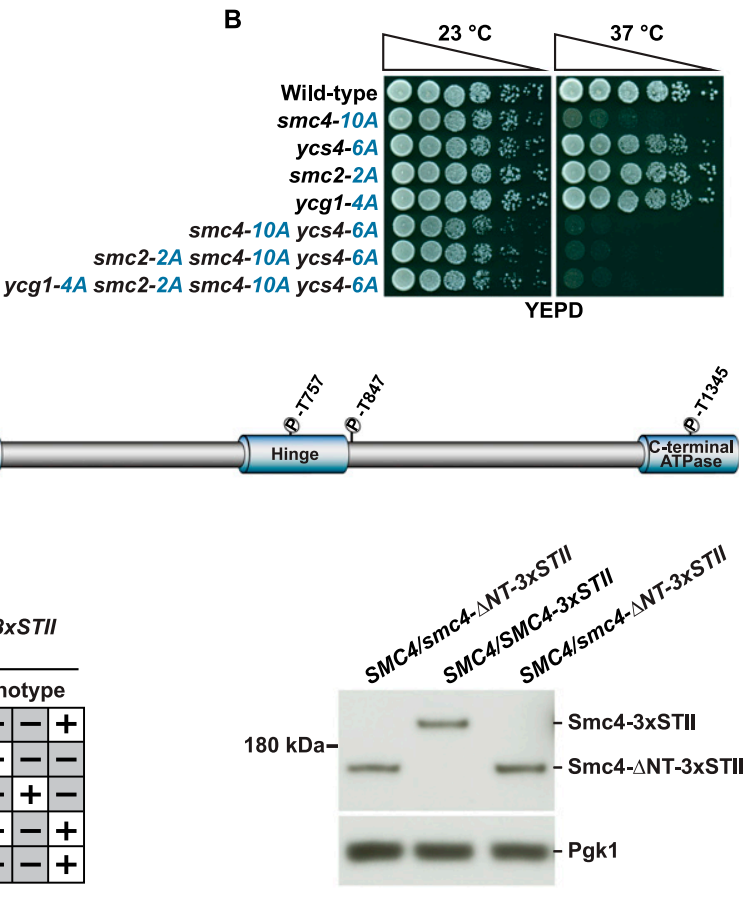

F

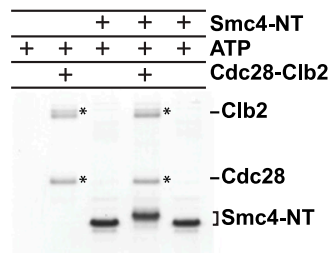

Figure 2. Smc4 is a key target of Cdk1 in the yeast condensin complex. (A) Schematic representation of the number of putative CDK sites (i.e., Ser/Thr-Pro) (Holt et al. 2009) in the subunits of the yeast condensin complex. $(B)$ Growth phenotype of condensin subunits lacking putative Cdk1 sites. Fivefold serial dilutions of the wild type and phospho-mutants were spotted on solid medium to evaluate growth at $23^{\circ} \mathrm{C}$ and $37^{\circ} \mathrm{C}$. (C) Schematic representation of Smc4 domain organization. The positions of Cdk1 phosphorylation sites are marked above the protein schematic. Sites identified by mass spectrometry are labeled with a white " $\mathrm{P}$," while other consensus Cdk1 sites were marked with a black "P." $(D)$ Deletion of the N-terminal extension of Smc4 is lethal in S. cerevisiae. Dissection of a sporulated heterozygous diploid strain carrying an allele of Smc4 that has lost its $\mathrm{N}$-terminal extension is shown at the left. The sequence of this particular allele starts at the position of the blue arrow in the sequence shown in Supplemental Figure S4C, which corresponds to the start site of other SMC family members. At the right, an immunoblot shows that loss of the N-terminal extension of Smc4 does not affect its protein abundance in heterozygous diploid cells. (E) In vitro kinase assay showing that Smc4 is a direct substrate for Cdk1. Purified condensin was incubated alone (first lane), with ${ }^{32} \mathrm{P}$-ATP (second lane), or in the presence of purified Cdc28-Clb2 complex and ${ }^{32}$ P-ATP (third lane). (Fourth lane) Purified histone $\mathrm{H} 1$ was used as positive control for Cdk1 phosphorylation. After incubation, proteins were separated on a $4 \%-12 \%$ SDS-polyacrylamide gel and revealed by Coomassie staining, as shown at the left. An autoradiograph of the same gel is shown at the right. Bullets indicate the different subunits of condensin. Asterisks correspond to the Cdk1-Clb2 complex, and the open circle marks the position of histone H1. $(F)$ The N-terminal extension of Smc4 is phosphorylated by Cdk1 in vitro. Purified Smc4-NT was incubated with ATP or purified Cdc28-Clb2 complex and ATP. After the kinase reaction, proteins were resolved by SDS-PAGE and stained with Coomassie.

(henceforth, Smc4-NT) to monitor in vivo phosphorylation. As expected, the electrophoretic behavior of Smc4-NT changes dramatically during the cell cycle, starting as a single band in G1 and acquiring at least two retarded species as cells progressed toward mitosis (Fig. 3A). Phosphatase treatment of Smc4-NT confirmed that the retarded species were due to phosphorylation (Fig. 3B). Importantly, Smc4-NT became phosphorylated simultaneously with or slightly prior to Swe1, an early Cdk1 substrate during mitosis (Harvey et al. 2005), and much earlier than Ycg1, a condensin subunit phosphorylated in anaphase (Fig. 3A; St-Pierre et al. 2009). These results indicate that Smc4 phosphorylation occurs at or very close to mitotic entry. Consistent with this interpretation, monitoring the phosphorylation of two of the Cdk1 sites-Ser4 and Ser128-on Smc4 using phospho-specific antibodies confirms that these residues are also modified early in mitosis (Fig. 3C,D; Supplemental Fig. S5A,B). The in vivo kinetics of Smc4 phosphorylation revealed by the Smc4-NT construct and the phospho-specific Ser128 antibody were essentially identical (Fig. 3D), thereby validating Smc4-NT as an effective reporter to monitor Smc4 phosphorylation status. Importantly, removal of the seven Cdk1 sites in the Smc4-NT fragment completely abrogated its phosphorylation-induced gel retardation in live cells (Fig. 3E; Supplemental Fig. S5C). These results strongly suggest that $\mathrm{Cdk} 1$ is the kinase that targets Smc4 for phosphorylation in early mitosis. Consistent with this prediction, cells defective in early (clb5 and clb6) and late (clb1, clb3, clb4, and clb2-ts) cyclin subunits showed marked reductions in the extent of Smc4 phosphorylation in vivo (Fig. 3F; Supplemental Fig. S5D,E). Moreover, removal of the Clb5 targeting RxL motifs (Loog and Morgan 2005; Koivomagi et al. 2011) in the $\mathrm{N}$ terminus of Smc4 caused a substantial reduction in the extent of its phosphorylation (Fig. 3G; Supplemental Fig. S5F). Finally, we wanted to test the possibility that Smc4 phosphorylation might be mediated by $\mathrm{Cdc} 5$, since this kinase is known to be activated by Cdc28 in mitosis (Mortensen et al. 2005). However, the fact that Smc4-NT phosphorylation remained normal in cdc5-99 mutant cells entering mitosis (Supplemental Fig. 

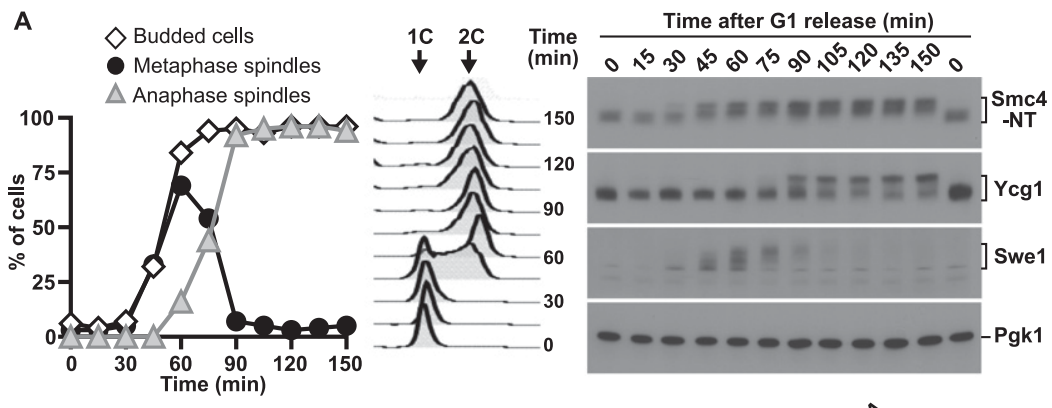

B

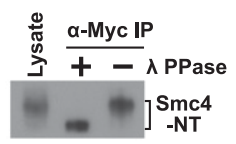

C

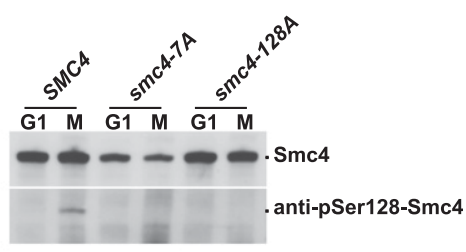

$E$
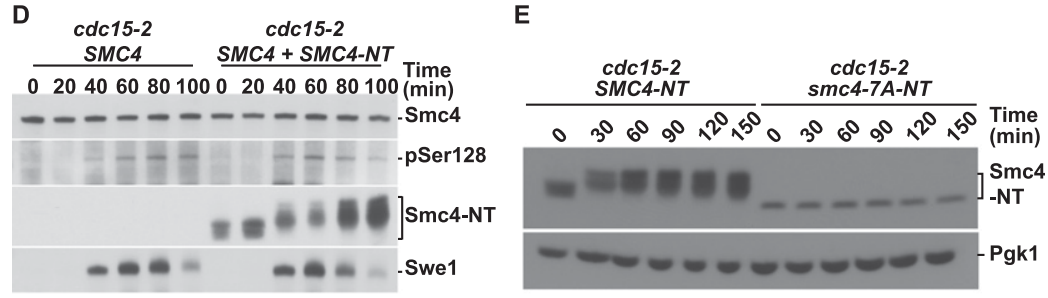

$\mathbf{F}$

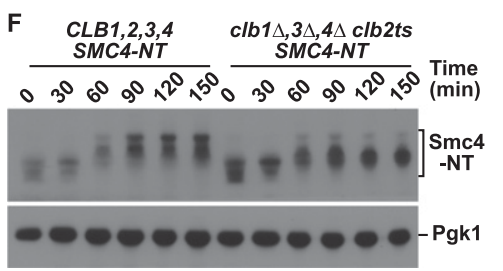

Figure 3. Cdk1 phosphorylates Smc4 in early mitosis. (A) Kinetics of Smc4 modification during the cell cycle. Cells expressing the Smc4-NT fragment were released from a G1 arrest into fresh medium at $37^{\circ} \mathrm{C}$. Samples were collected every $15 \mathrm{~min}$ and processed for visualization of cell morphology and mitotic spindle length (left panel), DNA content analysis (middle panel), and immunoblot analysis (right panel). Pgk1 serves as a loading control, while changes in the electrophoretic behavior of Swe1 and Ycg1 mark early and late mitotic events, respectively (Harvey et al. 2005; St-Pierre et al. 2009). (B) X-Phosphatase treatment of Smc4 isolated from mitotic cells. Following dephosphorylation, samples were processed as in $A .(C)$ Validation of antibodies against phospho-Ser128 (pSer128) of Smc4. Protein samples from interphase (G1) and mitotic (M) cells of the indicated genotypes were probed by immunoblot analysis for the presence of phosphorylated Smc4. (D) The appearance of the pSer128 signal parallels that of the phosphorylation-induced gel retardation of Smc4-NT during mitosis. cdc15-2 mutants carrying SMC4-3xSTII with and without the Smc4-NT fragment were released from a G1 arrest into fresh medium at $37^{\circ} \mathrm{C}$ and treated for immunoblot analysis as in $A$. The Smc4-3xSTII signal serves as a loading control, while the appearance of Swel marks mitotic entry, respectively. Note the concurrent appearance of the pSer128 and Swel signals as well as the gel retardation of Smc4-NT at $40 \mathrm{~min}$ after release. The budding index of the cultures is shown in Supplemental Figure S5A. $(E)$ Time course analysis of Smc4 phosphorylation in the presence and absence of Cdk1 sites. Cells expressing wild-type Smc4-NT or the Smc4-7A-NT phospho-mutant were released from a G1 block at $37^{\circ} \mathrm{C}$, and samples were taken every $30 \mathrm{~min}$ to evaluate phosphorylation-induced gel retardation. The spindle morphology of cells during the time course experiment is shown in Supplemental Figure S5C. $(F)$ Time course analysis of Smc4-NT phosphorylation in cells carrying $c l b 1 \Delta c l b 3 \Delta c l b 4 \Delta$ clb2-ts mutations. The experiment was performed as described in $E$, except that cells were released from G1 arrest in nocodazole-containing medium. The budding index of the cultures is shown in Supplemental Figure S5E. (G) Removal of RxL docking sites leads to a partial loss of Smc4 phosphorylation. cdc15-2 mutant cells expressing wild-type SMC4-NT or the smc4-rxl-NT mutant were released from a G1 block at $37^{\circ} \mathrm{C}$ and treated as in E. Percentages of budded cells are shown in Supplemental Figure S5F.

S5G) or in cells already arrested at this stage of the cell cycle (Supplemental Fig. S5H) argues against a role for this kinase as the major or sole Smc4 kinase in early mitosis. Taken together, our results show that effective phosphorylation of Smc4 in early mitosis requires direct targeting of condensin by cyclin-Cdk1 complexes.

\section{Smc4 phosphorylation activates chromosome morphogenesis}

What is the physiological significance of Smc4 phosphorylation by Cdk1? To answer this question, we introduced in the yeast genome mutations that either prevent or mimic the phosphorylation of the seven Cdk1 sites in the $\mathrm{N}$-terminal extension of Smc4. Whereas removal of individual Cdk1 sites resulted in little or no effect on growth properties and on chromosome condensation (Supplemental Fig. S6A,B), cells carrying a SMC4 allele that lacks all
$\mathrm{N}$-terminal Cdk1 sites exhibited a strong growth defect at the nonpermissive temperature (i.e., smc4-7A) (Fig. 4A). We noticed that the Smc4-7A protein was less abundant than its wild-type counterpart in immunoblot analysis (Fig. 4B, first two lanes). This reduction in Smc47A protein levels is not responsible for its growth defect, since down-regulation of wild-type Smc4 abundance using the auxin-inducible degron (i.e., Smc4-AID) did not result in detectable growth defects at Smc4 protein levels comparable with or lower than those of the Smc47A mutant (Fig. 4B,C, cf. $40 \mu \mathrm{M}$ auxin/IAA and smc4-7A lanes). Consistent with this, inactivation of the pathway responsible for the degradation of unstable nuclear proteins did not suppress the ts phenotype of smc4-7A cells (Supplemental Fig. S7A), and no defect in condensin complex formation was detected in smc4-7A mutants (Fig. 4D). Collectively, these results indicate that the conditional lethality phenotype of the smc4-7A mutant 
A

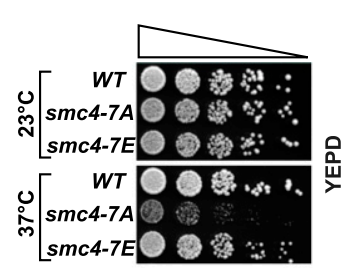

B

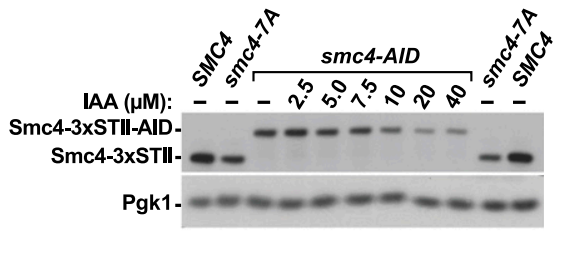

C
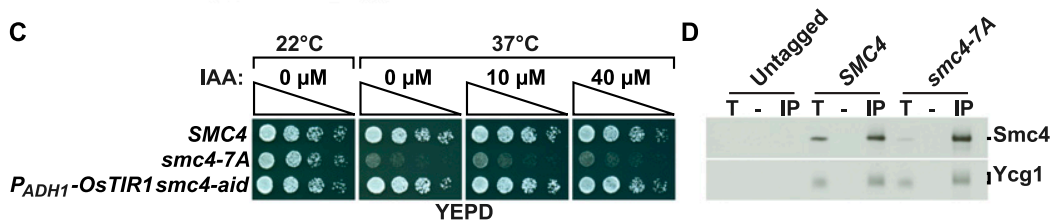

E
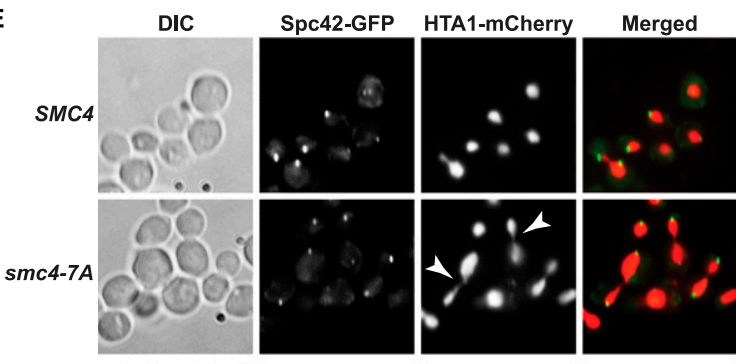

$\mathbf{F}$

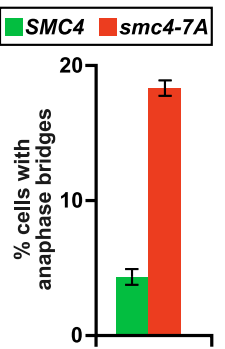

G

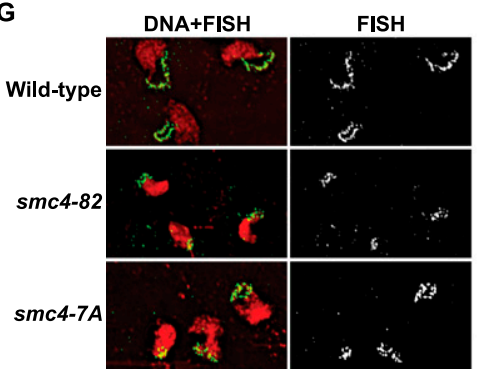

H

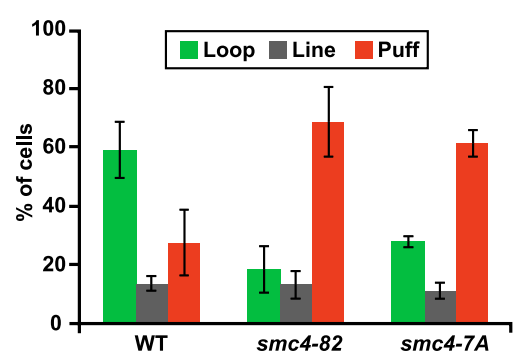

Figure 4. Cdk1-mediated phosphorylation of Smc4 is required for chromosome condensation. (A) Growth properties of phospho-mimetic and phospho-mutant forms of Smc4. Fivefold serial dilutions of smc4 phospho-mutants were spotted on solid medium to evaluate growth at $23^{\circ} \mathrm{C}$ and $37^{\circ} \mathrm{C}$. (B) Regulation of Smc4-AID protein abundance by auxin/IAA. Cells were grown asynchronously at $23^{\circ} \mathrm{C}$ until exponential phase and exposed to different concentrations of auxin for $2 \mathrm{~h}$ at $30^{\circ} \mathrm{C}$. Smc4-AID abundance was evaluated by immunoblot analysis using the $3 x$ StrepTagII epitopes at the $\mathrm{C}$ terminus of the protein. $(C)$ Effects of reduced Smc4-AID abundance on yeast growth properties. Strains carrying SMC4 or $s m c 4-7 A$ were included as controls to show normal and ts growth phenotypes in the presence of auxin. (D) The smc4-7A mutant is competent for condensin complex formation. Smc4 was immunoprecipitated from whole-cell extracts using anti-STII antibodies, and its ability to associate with Ycg1-3xFlag was determined by immunoblotting. Whole-cell extracts $(\mathrm{T})$ and beads only $(-)$ were loaded as controls. Approximately equal amounts of immunoprecipitated (IP) Smc4 were loaded in each lane to allow effective comparison of the levels of Ycg1 interacting with Smc4. $(E)$ smc4 phospho-mutants show cytological markers associated with chromosome segregation defects. A high proportion of anaphase nuclei connected with lagging genetic material (arrowheads) and having an elongated morphology can be seen in a culture of smc4-7A mutants growing at the nonpermissive temperature. Chromatin and spindle pole bodies were visualized using HTAl fused to mCherry and Spc42 fused to GFP, respectively. $(F)$ Quantification of anaphase bridges in the wild type and smc4 phospho-mutant $(n=3)$. Error bars

indicate SD. $(G)$ Chromosome condensation defects in the smc4 phospho-mutant. For each genotype, a representative micrograph of the most prominent rDNA species detected by FISH is shown. Cells were grown asynchronously at $23^{\circ} \mathrm{C}$ until exponential phase and shifted to $37^{\circ} \mathrm{C}$ for $2.5 \mathrm{~h}$. Nocodazole was used to block cells in metaphase. Nuclei and rDNA were stained with PI (red) and FITC (green), respectively. $(H)$ Quantification of rDNA condensation in SMC4, smc4-7A, and smc4-82 mutants grown at the nonpermissive temperature. At least 100 cells were counted for each genotype in three independent experiments. Error bars indicate SD.

is specifically due to the loss of Cdk1-mediated phosphorylation of condensin.

Microscopic examination of smc4-7A mutants at the nonpermissive temperature provided critical insights into the nature of the cellular defect responsible for the lethality of this mutant. We noticed that there was a significant fraction of dividing cells in the mutant population that contained unequal amounts of nuclear material and/or connecting threads of chromosomal DNA between separating nuclei (Fig. 4E, see arrowheads). This phenotype was much less frequent in wild-type cells (Fig. 4F) and is indicative of severe chromosome segregation defects in the absence of Smc4 phosphorylation. The root cause for the segregation defect of condensin mutants has been ascribed to their inability to promote chromosome condensation (Hirano 2012). To test this notion, we examined the rDNA condensation proficiency of the smc4-7A mutant and compared it with that of SMC4 and smc4-82 cells (i.e., a strong ts mutant of condensin) (see below). Whereas wild-type cells were able to condense the rDNA effectively at $37^{\circ} \mathrm{C}$, both the smc4-7A and smc4-82 mutants were completely defective in rDNA loop formation at nonpermissive temperatures (Fig. 4G,H). These results indicate that $\mathrm{Cdk} 1$ phosphorylation is absolutely necessary to activate condensin and initiate chromosome condensation in vivo.

A previous study using the fission yeast Schizosaccharomyces pombe suggested that $\mathrm{Cdc} 2$ might regulate condensin by phosphorylation. Sutani et al. (1999) showed that overexpression of the cut3-T19A phosphomutant caused a dominant-negative chromosome segregation defect in this organism (however, no chromosome condensation defects were observed in this study). Since the phenotype of the cut3-T19A mutant expressed at its natural level was not reported by Sutani et al. (1999) and 
protein overexpression can often obscure the physiological relevance of natural regulatory processes (Robbins and Cross 2010), we wondered what might be the phenotype associated with loss of Thr19 phosphorylation when Cut3 is expressed at physiological levels. To address this, we integrated the cut3-T19V phosphomutant at its natural locus in diploid $S$. pombe. The valine substitution was used in cut 3 instead of alanine, since valine is structurally more similar to threonine, thus precluding destabilizing effects not related to loss of phosphorylation. Dissection of a sporulated heterozygous diploid yeast carrying the cut3-T19V allele revealed that loss of Thr19 phosphorylation is lethal in haploid cells when the mutant is expressed from its physiological locus (Supplemental Fig. S7B). Interestingly, the heterozygous diploid carrying cut3-T19V grew normally (Supplemental Fig. S7C), thereby suggesting that the dominant-lethal effects previously observed with the cut3-T19A mutant might be specific to the alanine substitution and/or the overexpression of the protein (Sutani et al. 1999). Taken together, these results further strengthen the conclusion that Cdk1 phosphorylation of the $\mathrm{N}$-terminal extension of Smc4 family members plays a critical role in the regulation of condensins in eukaryotes.

\section{Cdk1 phosphorylation sites activate condensin by altering its charge}

We next examined the phenotype of a SMC4 allele carrying phospho-mimetic residues (i.e., negatively charged glutamates) at the position of Cdk1 sites. Constitutive activation of condensin by such mutations is expected to create a lethal form of the enzyme because it would permanently alter chromatin structure in yeast. However, replacement of phosphorylated residues by glutamates in Smc4 did not result in an obvious activation of condensin or a strong growth defect in the smc4-7E mutant (Fig. 4A). This is not completely unexpected, since a single negatively charged residue does not accurately mimic the structure or net charge of a phosphorylated residue, as previously observed (Lyons and Morgan 2011; Pearlman et al. 2011). Based on the fact that the Nterminal extension of Smc4 is predicted to be highly unstructured (Supplemental Fig. S8A-C), we considered the possibility that the change in charge imparted by phosphorylation of this domain would be more relevant as a regulatory mechanism than a possible change in structure. We therefore created a smc4-7EE allele where the phosphorylated residues and adjacent prolines $1+1$ position of the CDK motif) were mutated to double glutamates to more accurately mimic phosphate charges (Fig. 5A,B; Pearlman et al. 2011). It was recently shown that this approach is more effective at mimicking an "activated" state than single glutamate/aspartate substitutions in substrates targeted by proline-directed kinases (Strickfaden et al. 2007; Li et al. 2014). The charge-mimetic allele, together with additional control mutations, was integrated at the SMC4 locus in a diploid yeast strain, and the resulting heterozygous mutants were sporulated and dissected to reveal the phenotypes of the mutations in haploid spores. Remarkably, introducing dual charge-mimetic mutations at the seven phospho-sites and adjacent positions (i.e., Ser/Thr-Pro motif) of Smc4 led to lethality in all spores inheriting those mutations, whereas simultaneously altering the seven phospho-sites with single glutamates at either position of the Ser/Thr-Pro motifs had no detectable effect on viability (i.e., four viable spores per tetrad) (Fig. 5B). Moreover, a mutant carrying dual glutamines-an amino acid approximately isosteric with glutamate but lacking the negative charge (Fig. 5A) - did not show any obvious viability defects (Fig. 5B). These results demonstrate that the lethality of the smc4-7EE mutant is due to the presence of negative charges that effectively mimic the phosphorylated state of the active protein. Immunoblot analysis of heterozygote diploids confirmed that the Smc4-7EE mutant is expressed at normal levels in yeast (Supplemental Fig. S8D).

To further strengthen our previous interpretation, we asked whether an alternative means of imposing a constitutive state of phosphorylation on Smc4 would recapitulate the phenotype of the smc4-7EE allele. To achieve this, we fused the B-type cyclin Clb2 to the $\mathrm{N}$ terminus of Smc4 in order to target Cdk1 to Smc4 throughout the cell cycle, an experimental approach previously described (Lyons and Morgan 2011). Dissecting a sporulated heterozygous diploid strain carrying the CLB2-SMC4 fusion gene led to lethality or severe growth defects in most of the spores inheriting the fusion construct (Fig. 5B). Preventing phosphorylation of Smc4 by removal of its phospho-sites or deletion of Clb2's cyclin box (the part of the protein responsible for interaction with Cdk1) largely suppressed the lethality associated with the cyclin-Smc4 fusion protein (Fig. 5B; Supplemental Fig. S9A). These results are consistent with the phenotype of the smc4$7 E E$ allele and indicate that inducing a permanent state of phosphorylation in Smc4 is incompatible with viability in yeast. Since the phenotype associated with mimicking phosphorylation is much stronger than that caused by loss of phosphorylation, we posit that the smc4-7EE and CLB2SMC4 mutants behave as gain-of-function alleles in vivo.

We envision two possible regulatory modes that can account for the lethality of the smc4-7EE and CLB2SMC4 mutants. First, constitutive phosphorylation of condensin may simply activate the enzyme and result in a permanent state of chromosome condensation that is not compatible with viability. Alternatively, preventing phosphate removal in phospho-mimetic mutants may abrogate iterative phosphorylation/dephosphorylation cycles that are normally required to maintain full enzyme activity during mitosis (De Wulf et al. 2009). Overexpression of smc4-7EE can distinguish between these two regulatory modes, since a version of condensin that is fully activated by static phosphorylation (i.e., no turnover model) would be expected to induce unscheduled condensation following overexpression, whereas a version of this enzyme that is engaged into its activation cycle by phosphorylation but not allowed to complete it by dephosphorylation (i.e., dynamic phosphorylation model) would act as a dominant-negative inhibitor. To test these hypotheses, we expressed smc4-7EE ectopically in wild- 


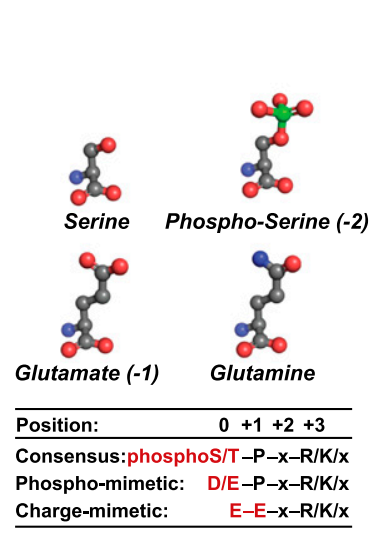

C
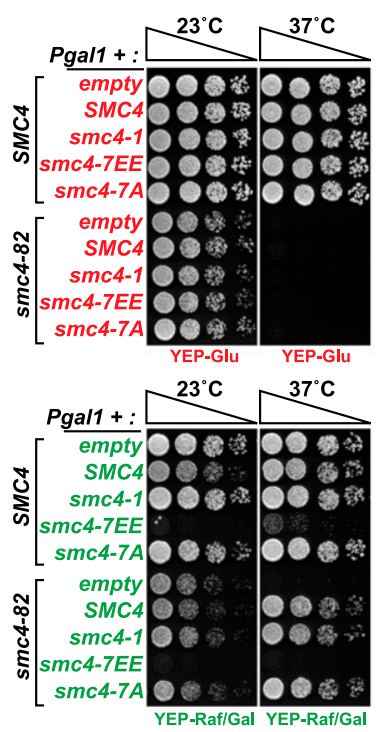

B

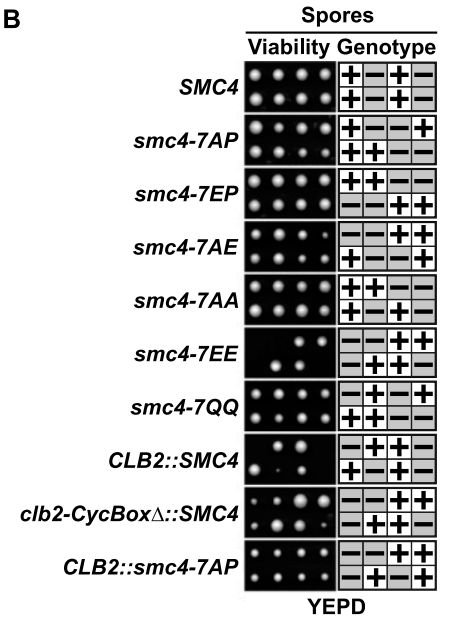

D

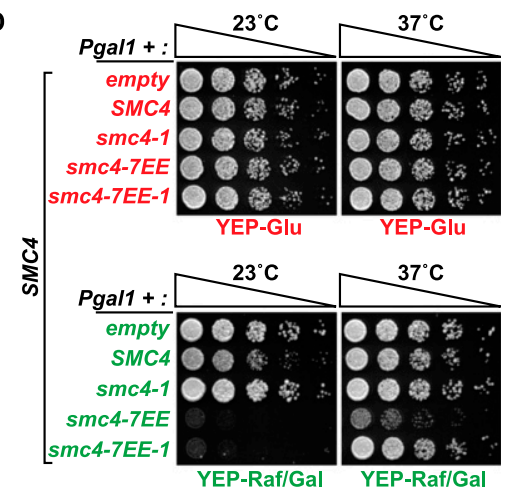

E

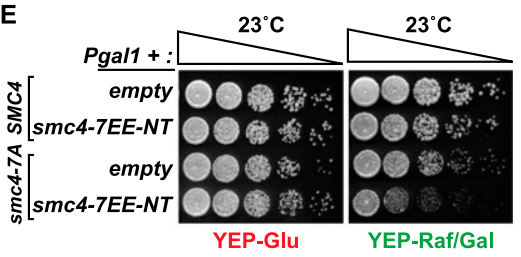

Figure 5. Constitutive phosphorylation creates a dominant-negative form of Smc4. (A) Structural basis of phospho-mimicking mutations in SMC4. (Top) Ball-and-stick representation of different amino acids used to substitute the phosphorylated residues in Smc4. (Bottom) Positions of the mutagenic substitutions used in this study relative to the Cdk1 optimal consensus for phosphorylation. $(B)$ Physiological effect of charge-mimetic mutations in SMC4. Diploid strains carrying smc4 phospho-mimetic, charge-mimetic, and phospho-constitutive mutations were induced to sporulate, and the viability of the resulting haploid spores was determined after $3 \mathrm{~d}$ of growth on solid medium. Two typical tetrads of spores are shown per genotype. The genotype of spores was ascertained using the HIS 3 marker associated with the mutant alleles of SMC4 (i.e., + sign means wild-type SMC4). Phospho-defective substitutions are included as controls. $(C)$ Overexpression of smc4-7EE leads to dominant lethality in cells. Fivefold dilution series of the wild type and smc4-82 mutant harboring galactose-inducible versions of SMC4 integrated at the URA3 locus were spotted on solid medium to evaluate growth at $23^{\circ} \mathrm{C}$ and $37^{\circ} \mathrm{C}$. Unlike smc4-1 or smc4-7A alleles, expression of smc4-7EE induced strong dominant-negative lethality irrespective of the genetic background or temperature used for overexpression. $(D)$ The smc4$7 E E$ allele encodes a gain-of-function mutant. Fivefold dilution series of wild-type cells expressing a galactose-inducible smc4-7EE-1 chimeric mutant (or SMC4, smc4-1, or smc4-7EE controls) integrated at the URA3 locus were spotted on solid medium to evaluate growth as in $C$. $(E)$ The charge-mimetic mutations can act in trans. Wild-type or smc4-7A mutant cells expressing a galactose-inducible smc4$7 E E-N T$ construct were spotted on solid medium, as in $C$. type and clb2-ts-arrested cells. Remarkably, ectopic expression of $s m c 4-7 E E$ was not able to induce unscheduled condensation of the rDNA locus in G1 cells or full rDNA condensation in G2/early mitotic cells (Supplemental Figs. S9B-E, S10A), which suggests a need for dynamic phosphorylation in the regulation of condensin in vivo. Consistent with this, overexpression of smc4-7EE led to dominant-negative lethality in both the wild type and smc4-82 mutants (Fig. 5C). This lethality is not due to unspecific loss of function in smc4-7EE or subunit imbalance in condensin, since overexpression of smc4-1 (Freeman et al. 2000), smc4-7A, or SMC4 complemented the lethality of the smc4-82 mutant at $37^{\circ} \mathrm{C}$ without causing dominant-lethal effects in wild-type cells (Fig. $5 \mathrm{C})$. However, we note that overexpression of SMC4 in wild-type cells caused a weak proliferation delay at $23^{\circ} \mathrm{C}$.

It had been previously demonstrated that the effects of mutations that confer a gain of activity on a protein can be abrogated by fusion with known inactivating muta- tions (Schott and Hoyt 1998). To formally test the gain-offunction nature of the charge-mimetic mutations in SMC4, we combined the 7EE substitutions with the inactivating mutations in the smc4-1 allele and monitored the ability of the chimeric mutant to behave in a dominant-negative manner. As expected, overexpression of the smc4-7EE-1 chimeric mutant in conditions under which its charge-mimetic mutations are not counteracted by the smc4- 1 mutations (i.e., $23^{\circ} \mathrm{C}$, the permissive temperature for smc4-1) led to a dominant-negative lethality similar to that of the smc4-7EE single mutant (Fig. 5, D vs. C,E). In contrast, overexpression of the chimeric mutant at $37^{\circ} \mathrm{C}$ (i.e., the inactivating condition for smc4-1 and, by extension, smc4-7EE) suppressed the lethal gain of function associated with the charge-mimetic substitutions (Fig. 5D). Since Smc4-7EE must be endowed with an activity to be able to lose it, we conclude from this experiment that the charge-mimetic mutations impart a new activity on Smc4 that requires 
otherwise normal protein function to mediate its effect. Importantly, this new activity can be induced equally well by fusion of SMC4 with CLB2 or by charge-mimicking mutations, which indicates that Cdk1 phosphorylation activates condensin by altering its charge in living cells and that preventing Smc4 dephosphorylation is lethal in vivo. Consistent with this interpretation, inactivation of a major mitotic phosphatase and known condensin interactor, PP2A (encoded by the PPH3, PPH21, and PPH22 genes in budding yeast) (Yeong et al. 2003; Takemoto et al. 2009; Peplowska et al. 2014), caused a large reduction in the ability of phosphatasedefective cells to maintain chromosome condensation in metaphase (Supplemental Fig. S10B). Collectively, our results indicate that dynamic turnover of Cdk1 phosphorylation events on condensin is important for mitotic chromosome condensation.

\section{Hypersensitivity of Smc4 to Cdk1 phosphorylation}

How might charge-driven activation of condensin promote early mitotic appearance of condensed chromosomes? One likely possibility would be that condensin is hypersensitive to low levels of Cdk1 activity because phosphorylation of any individual Cdk1 site is functionally equivalent for enzyme activation, and only a few of them need to be modified (in any combination) to fully activate the enzyme. The dominant effects of chargemimetic mutations provide a unique means to test this hypothesis. Indeed, one would expect that progressive removal of the seven dual glutamates in Smc4 would eventually reverse the partial gain of function of the smc4-7EE allele, thereby revealing the minimal number of phospho-sites that is required to activate condensin in vivo.

Prompted by this rationale, we created a series of heterozygous SMC4/smc4-EE mutants carrying progressively smaller numbers of dual-glutamate mutations and tested their phenotypes after sporulation of diploid cells. Remarkably, diploid strains carrying as few as three to four charge-mimetic mutations gave rise to tetrads with a very penetrant 2:2 lethality phenotype cosegregating with smc4 mutations (Fig. 6, rows 5,6). This is identical to the phenotype of the original smc4-7EE or smc4-10EE alleles (Fig. 6, rows 2,3) but is in contrast to the absence of phenotype of mutations affecting the first three Cdk1 sites at the $\mathrm{N}$ terminus of the protein (Fig. 6, row 4). This result suggests that three to four phosphorylation events in the middle or C-terminal clusters of Cdk1 sites may be sufficient to activate Smc4. To further refine this conclusion, we focused our analysis of charge-mimetic mutations on the middle cluster of phospho-sites (i.e., positions 109, 113, 117, and 128). Removing a single dualglutamate mutation from the four present in this cluster did not fully suppress the lethality of the remaining charge-mimetic mutations (Fig. 6, rows 7,8). However, loss of any additional site either reduced the penetrance of the lethality phenotype (Fig. 6, row 9) or allowed complete recovery of spore viability (Fig. 6, rows 10-14). Importantly, preventing phosphorylation of the Cdk1 sites away from the middle cluster of dual glutamates (i.e., the smc4-3A-4EE-3A mutant) did not suppress the effect of the smc4-4EE mutant (i.e., Fig. 6, cf. rows 5 and 17), thereby indicating that the lethality of this strain was not due to phosphorylation of the remaining Cdk1 sites in Smc4-4EE. Taken together, these results indicate that a threshold of two to three phospho-mimicking charges is sufficient to generate dominant condensin activity in vivo (Fig. 6, rows 7,16). Moreover, our data reveal a degree of functional equivalency (or redundancy) in Cdk1 phospho-sites, thereby explaining how condensin activation in early mitosis can be hypersensitive to Cdk1 levels.

\section{Cdk1 controls condensin binding to chromatin}

Finally, we wished to identify the specific aspect of condensin behavior that was regulated by Cdk1 phosphorylation. Multiple events have been previously shown to be required for full activation of condensin in eukaryotes, including nuclear import (Sutani et al. 1999), recruitment on chromatin (Freeman et al. 2000), and enzymatic activation (Kimura et al. 1998). The fact that budding yeast condensin and mammalian condensin II are constitutively located in the nucleus (Freeman et al. 2000; Ono et al. 2004; Gerlich et al. 2006) suggests that the Cdk1 regulation of these enzymes may not occur at the level of nuclear import. Interestingly, previous studies in human cells showed that condensin II binding to chromatin is very dynamic prior to chromosome condensation but then becomes much more stable as cells enter mitosis (Gerlich et al. 2006). The mechanistic basis for this change in chromatin-binding dynamics is unknown, but it seems likely that increasing the duration of condensin interactions with chromatin would stimulate chromosome condensation. To evaluate whether condensin phosphorylation by Cdk1 could affect its chromatin-binding dynamics, we used photobleaching confocal laser-scanning microscopy. A subdomain in the nucleus of live yeast cells expressing GFP-fused Smc4 was photobleached using the line scan feature of the microscope, and the kinetics of fluorescence decay were determined over time at the region of interest in the cell (i.e., red rectangle in Fig. 7A). In this experiment, the Smc4-3xGFP contained within the unbleached area is expected to replenish the fluorescence in the photobleached area of the cell at a rate that is directly proportional to the exchange of condensin complexes between the two regions within the nucleus. As expected, preventing exchange by fixation of cells with paraformaldehyde led to a very rapid loss of Smc4-3xGFP fluorescence signal in the photobleached area while leaving the fluorescence in the rest of the nucleus unaffected (Fig. 7A). In contrast, photobleaching a subdomain of the Smc4-3xGFP signal in live cells resulted in a rapid decay of fluorescence signal throughout the entire yeast cell (Fig. 7A,B). This rapid loss of fluorescence is consistent with the highly dynamic exchange of condensin complexes between the site of photobleaching and the rest of the nucleus, a behavior consistent with that of condensin I in higher eukaryotes (Gerlich et al. 2006). Remarkably, performing the same experiment in cells expressing Smc4-7A revealed that the 


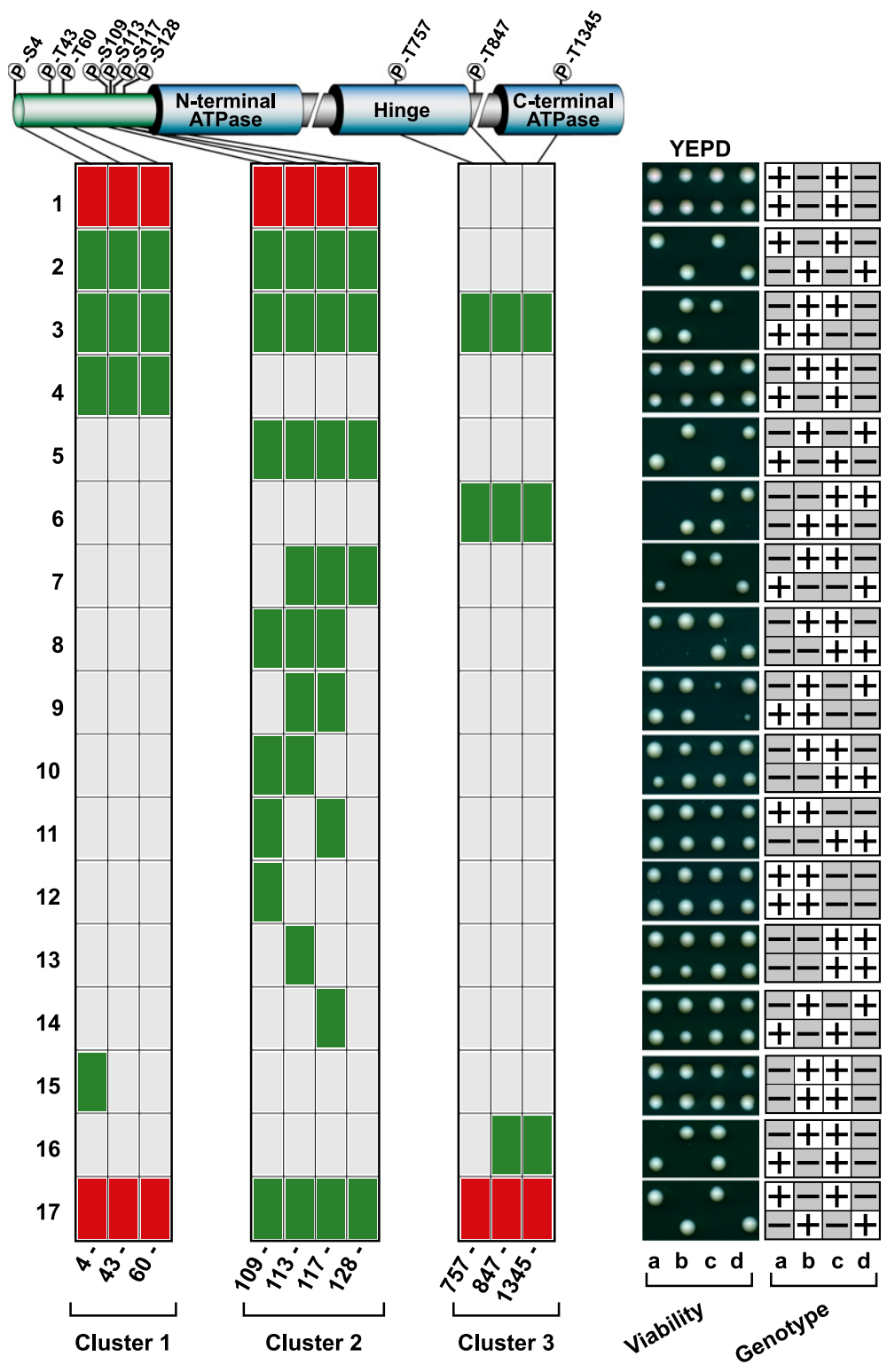

Figure 6. Determination of the minimal number of charge-mimetic mutations required to create a dominant form of condensin. A schematic representation of Smc4 domains depicting the positions of the phosphorylated residues modified in this analysis is shown at the top. The combinations of dual charge-mimetic substitutions used in the various mutants are shown in the chart at the left. Phosphorylated residues are represented in the chart by small rectangles that are color-coded according to their mutagenic status (i.e., gray is wild-type, red corresponds to single alanine, and green represents dual charge-mimetic). The viability and genotypes of two typical tetrads of spores resulting from the dissection of diploid strains carrying the various smc4 mutations are shown at the right of the chart.

\section{= Phosphomutant}

= Charge-mimetic

= Wild-type fluorescence decay of the phospho-mutant is significantly slower than that of the wild-type protein (Fig. 7B). This result is consistent with Smc4-3xGFP residing in the bleaching volume for longer periods of time while it is bound to chromatin, thereby causing it to bleach more quickly. The rapidly exchanging Smc4-7A still binds to DNA but does not reside in the bleaching region as long, so it takes a longer time to photobleach. To quantify this difference, decay curves were fit with a two-component exponential decay model with a short $(d)$ and long $(b)$ time decay constant (cf. Equation 1 in the Supplemental Material). Both decay constants were significantly longer for the highly dynamic Smc4-7A or cytosolic GFP protein than that of wild-type Smc4 $(P \leq 0.001)$ (Fig. 7B,C; decay constant $d$ is shown in Supplemental Fig. S11A). Taken together, these results reveal that Cdk1 phosphorylation extends the duration of condensin's interaction with chromatin, thereby providing an explanation of how this post-translational modification regulates chromosome condensation during mitosis. These rapid protein dynamics in the yeast nucleus are consistent with other studies done using fluorescence correlation spectroscopy in this organism (Slaughter et al. 2007).

\section{Discussion}

Quantitative activation of chromosome condensation in early mitosis

The central prediction from the quantitative model of Cdk1 action is that cellular events occurring early in the cell division program must be more readily activated by Cdk1 
Robellet et al.

A

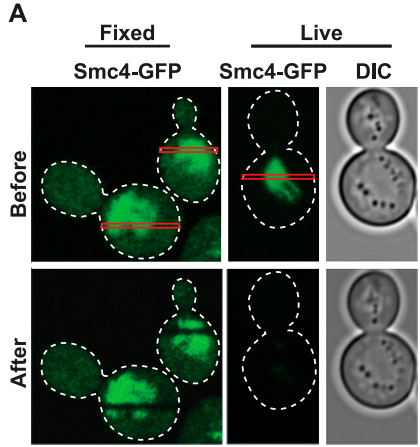

C

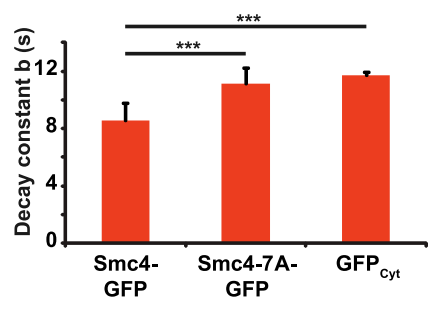

B

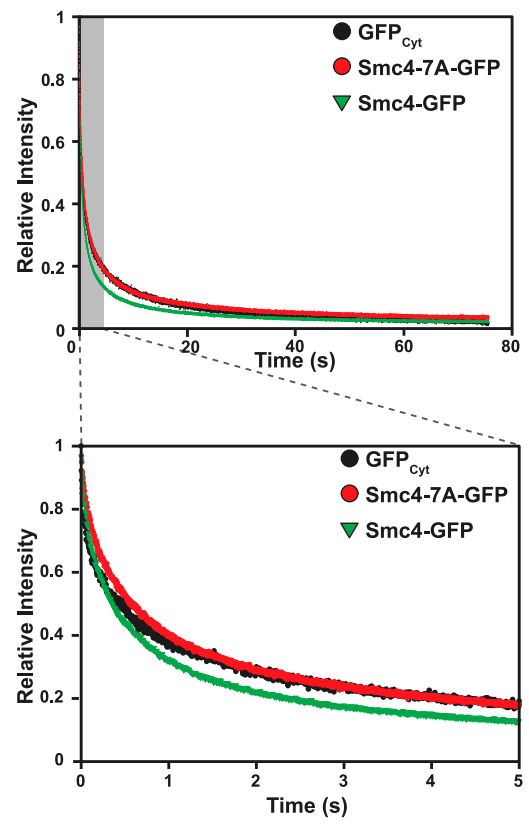

D
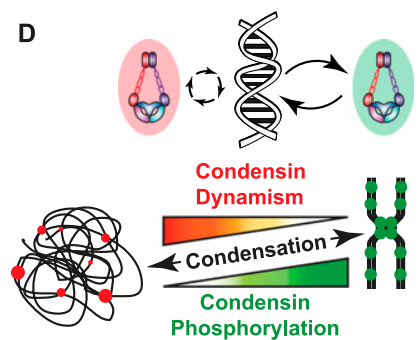

Phosphorylation

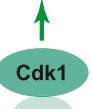

СусB
Figure 7. Cdk1 phosphorylation regulates the dynamics of condensin interaction with chromatin. $(A)$ Decay of fluorescence after photobleaching of cells expressing Smc4-3xGFP. Images were collected, and photobleaching was performed using a $63 \times / 1.4 \mathrm{NA}$ plan-apochromat oil immersion objective lens. Single DIC and fluorescence images of cells in metaphase were collected before and after each photobleaching experiment. Photobleaching was performed using 20,000 line scans of 512 pixels across the center of the cell with a $488-\mathrm{nm}$ laser at $20 \%$ power. Emission from the sample was collected from $493 \mathrm{~nm}$ to $598 \mathrm{~nm}$. Photobleaching was performed on fixed cells (left) or live cells (right). The top panels show Smc4-3xGFP fluorescence prior to treatment, whereas the bottom panels show remaining fluorescence after line scan photobleaching. The region of interest in each cell is labeled with a red rectangle, whereas the cell outline is labeled with a dashed line. DIC micrographs are shown for live cells. (B) Graphs representing the kinetics of GFP fluorescence decay over time after line scan photobleaching of cells expressing SMC4$3 \times G F P$, the smc4-7A-3xGFP mutant, or cytosolic GFP. Details of the data analysis are in the Materials and Methods section. The bottom graph shows data corresponding to the first 5 -sec interval of the experiment. $(C)$ Histogram showing the calculated decay constant $b$ of wild-type Smc4, the Smc4-7A mutant, and cytosolic GFP. Triple asterisks indicate a significant difference in decay constant for cells expressing different versions of Smc4-3xGFP $\left(\left[{ }^{\star \star \star}\right]\right.$ $P<0.001)$. Error bars indicate SD over three independent experiments ( $n \geq 12$ cells per experiment). (D) Model for the role of Cdk1 in the regulation of chromosome morphogenesis (see the text for details). than later events of the cell cycle (Stern and Nurse 1996). Although differential susceptibility to Cdk1 activation explains the directionality of $S$ and $M$ phases (Coudreuse and Nurse 2010), the notion that a similar process would also explain the specific order of events in the mitotic program is currently unknown. Here, we show that initiation of chromosome condensation can be achieved at levels of Cdk1 activity that are too low to activate other mitotic processes. This result demonstrates that the order of cellular events in early mitosis is dictated by quantitative differences in substrate susceptibility to Cdk1 activation. Mechanistically, we show that the temporal kinetics of chromosome condensation in mitosis can be explained by functional redundancy in the phosphorylation sites that Cdk1 uses to activate condensin in vivo. Multisite redundancy in phospho-regulatory events is known to enable quantitative activation of effectors at very low levels of kinase activity in signaling cascades (Ferrell 1996).

\section{Identification of a novel chromosome-folding state in early mitosis}

The nature of the individual steps leading to the formation of condensed mitotic chromosomes has been a topic of much debate over the years. Previous attempts to define the architecture of mitotic chromosomes have been frustrated by the absence of cytologically defined chromatin-folding intermediates in the process of condensation. More recently, a study has proposed a two-stage mechanism of chromosome condensation that involves longitudinal compression of arrays of consecutive chromatin loops (Naumova et al. 2013). This model was generated using in silico polymer simulations and predicts that the formation of local loops of chromatin would represent the initial stage of chromosome condensation. Our identification of rDNA condensation intermediates containing intertwined loops of thin chromatin threads-the intertwist configuration-gives credence to this in silico model. Moreover, the resolution of the intertwist configuration into a thicker thread of rDNA chromatin later in mitosis indicates that intertwined loops of rDNA are eventually resolved by compaction into a linear organization in yeast, as previously suggested for human chromosomes (Naumova et al. 2013). Taken together, our results provide the first direct observation of a two-step process involving distinct chromatin-folding states as intermediates in the process of chromosome condensation. 
Mechanistic basis for condensin activation in early mitosis

Our study reveals that the unique $\mathrm{N}$-terminal extension of Smc4 family members acts as a modulator of condensin's ability to bind on chromatin. Upon phosphorylation by Cdk1, condensin binding to the bulk of chromatin becomes less dynamic relative to the unphosphorylated enzyme and thus allows extended interactions with mitotic recruiters to promote condensation (Fig. 7D; Supplemental Fig. S11B). It is tempting to speculate that Cdk1 phospho-sites on Smc4 could enhance-by virtue of their negative charges-the stability of the interaction between condensin and the positively charged residues in the N-terminal tails of histone $\mathrm{H} 2 \mathrm{~A}$ and $\mathrm{H} 2 \mathrm{~A} . \mathrm{Z}$ on chromosomes (Tada et al. 2011). Such stabilization would nicely explain the observed Cdk1-dependent reduction in the dynamic binding of condensin to chromatin in yeast and human cells (Gerlich et al. 2006). Importantly, this model explains how condensin can associate with chromatin throughout interphase without actually inducing chromosome condensation before mitosis (Freeman et al. 2000).

From an evolutionary perspective, the mechanisms that we unraveled in budding yeast are likely to be conserved in a wide range of eukaryotes. Notably, the Cdk1 consensus sites within the $\mathrm{N}$-terminal extension of Smc4 homologs are modified by phosphorylation in several organisms, including fission yeast, budding yeast, and humans (Supplemental Fig. S4C; Bazile et al. 2010). We envision that these Smc4 phosphorylation events collaborate with other regulatory events, such as Aurora B, Polo kinase, and Cdk1 phosphorylation of non-SMC subunits, to establish a chromosome architecture that is sufficiently condensed and yet adaptable to the unique cellular conditions experienced during mitosis (Bazile et al. 2010; Hirano 2012). In particular, it is conceivable that the appearance of a second condensin complex during evolution has resulted in a "division of labor" and sharing of Cdk1 phospho-sites between Smc4 and CapD subunits (Supplemental Fig. S12) to allow a regulation that is highly specific to either condensin I or II complexes. In organisms where condensin is monomorphic, such as yeast, current data indicate that this regulation would occur solely at the level of Smc4. This simplified regulation has enabled us to reveal how Cdk1 can act as an essential and high-sensitivity trigger for the initiation of chromosome morphogenesis during mitosis.

\section{Materials and methods}

\section{Yeast genetics and molecular biology}

All S. cerevisiae strains used in this study are isogenic with K699 and K700. Yeast growth conditions, medium composition, and procedures for genetic analysis were as described previously (Guthrie and Fink 1991). The genotypes of yeast strains used in this study are listed in Supplemental Table 1. Additional details relating to mutant yeast construction and molecular biology procedures are also included in the Supplemental Material.

\section{Microscopy}

For fluorescence in situ hybridization (FISH) analyses, cells were fixed in $0.1 \mathrm{M} \mathrm{KPO}_{4}$ buffer (pH 6.4) containing 3.7\% formaldehyde for $2 \mathrm{~h}$ at $23^{\circ} \mathrm{C}$. Probe preparation and hybridization were performed as described previously (Guacci et al. 1994; Lavoie et al. 2004). Images of rDNA morphology were acquired on a DeltaVision microscope equipped with the softWoRx software (Applied Precision). For photobleaching experiments, cells were grown at $25^{\circ} \mathrm{C}$, and images were acquired on a Zeiss 710 confocal laser-scanning microscope (Carl Zeiss) after photobleaching. See the Supplemental Material for a detailed description of specific conditions used for microscopy experiments.

\section{Electrophoresis and immunoblotting}

Eight percent Phos-tag acrylamide gels (Wako Chemicals USA) were used to resolve Smc4-NT phosphorylated species (Kinoshita et al. 2006), whereas gels containing 7.5\% Next gel acrylamide (Amresco) were used to monitor Smc4 abundance and Ycg1 phosphorylation (St-Pierre et al. 2009). All gels were transferred using the iBlot apparatus (Invitrogen). Antibodies and conditions used for immunoblotting are listed in the Supplemental Material.

\section{Mass spectrometry analysis of Smc4 phosphorylation}

To identify the Cdk1 phosphorylation sites on Smc4, condensin was immunopurified from protein extracts prepared from cells arrested in metaphase using nocodazole. Liquid chromatography-tandem mass spectrometry (LC-MS/MS) analysis was performed on immunoprecipitated condensin, as described previously (St-Pierre et al. 2009). An extended description of all methods used in this study is provided in the Supplemental Material.

\section{Acknowledgments}

We thank Julie St-Pierre and members of the D'Amours laboratory for their comments on the manuscript; Adam Rudner, Pascal Bernard, Alain Verreault, Angelika Amon, Michael Stark, and Fred Cross for antibodies and/or yeast strains; Martin Audet for Figure 5A design; and David Morgan for communicating unpublished results. Research in D.D.'s laboratory is supported by grants from the Canadian Institutes of Health Research (CIHR; MOP-136788, MOP-82912) and the Canadian Cancer Society Research Institute (CCSRI; 20304). X.R. is supported by a fellowship from the Cole Foundation. D.D. is recipient of a Canada Research Chair in Cell Cycle Regulation and Genomic Integrity. Photobleaching experiments were performed in the McGill Life Sciences Complex Advanced BioImaging Facility (ABIF).

\section{References}

Abe S, Nagasaka K, Hirayama Y, Kozuka-Hata H, Oyama M, Aoyagi Y, Obuse C, Hirota T. 2011. The initial phase of chromosome condensation requires Cdk1-mediated phosphorylation of the CAP-D3 subunit of condensin II. Genes Dev 25: 863-874.

Amon A, Tyers M, Futcher B, Nasmyth K. 1993. Mechanisms that help the yeast cell cycle clock tick: G2 cyclins transcriptionally activate G2 cyclins and repress G1 cyclins. Cell 74: 993-1007.

Baxter J, Aragon L. 2012. A model for chromosome condensation based on the interplay between condensin and topoisomerase II. Trends Genet 28: 110-117. 
Bazile F, St-Pierre J, D'Amours D. 2010. Three-step model for condensin activation during mitotic chromosome condensation. Cell Cycle 9: 3243-3255.

Bishop AC, Ubersax JA, Petsch DT, Matheos DP, Gray NS, Blethrow J, Shimizu E, Tsien JZ, Schultz PG, Rose MD, et al. 2000. A chemical switch for inhibitor-sensitive alleles of any protein kinase. Nature 407: 395-401.

Cimini D, Mattiuzzo M, Torosantucci L, Degrassi F. 2003. Histone hyperacetylation in mitosis prevents sister chromatid separation and produces chromosome segregation defects. Mol Biol Cell 14: 3821-3833.

Coudreuse D, Nurse P. 2010. Driving the cell cycle with a minimal CDK control network. Nature 468: 1074-1079.

De Wulf P, Montani F, Visintin R. 2009. Protein phosphatases take the mitotic stage. Curr Opin Cell Biol 21: 806-815.

Ditchfield C, Johnson VL, Tighe A, Ellston R, Haworth C, Johnson T, Mortlock A, Keen N, Taylor SS. 2003. Aurora B couples chromosome alignment with anaphase by targeting BubR1, Mad2, and Cenp-E to kinetochores. I Cell Biol 161: 267-280.

Ferrell JE Jr. 1996. Tripping the switch fantastic: how a protein kinase cascade can convert graded inputs into switch-like outputs. Trends Biochem Sci 21: 460-466.

Flemming W. 1882. Zellsubstanz, Kern und Zelltheilung. F.C.W. Vogel, Leipzig, Germany.

Freeman L, Aragon-Alcaide L, Strunnikov A. 2000. The condensin complex governs chromosome condensation and mitotic transmission of rDNA. J Cell Biol 149: 811-824.

Gavet O, Pines J. 2010. Progressive activation of CyclinB1-Cdk1 coordinates entry to mitosis. Dev Cell 18: 533-543.

Gerlich D, Hirota T, Koch B, Peters JM, Ellenberg J. 2006. Condensin I stabilizes chromosomes mechanically through a dynamic interaction in live cells. Curr Biol 16: 333-344.

Gong D, Ferrell JE Jr. 2010. The roles of cyclin A2, B1, and B2 in early and late mitotic events. Mol Biol Cell 21: 3149-3161.

Guacci V, Hogan E, Koshland D. 1994. Chromosome condensation and sister chromatid pairing in budding yeast. J Cell Biol 125: $517-530$.

Guthrie C, Fink GR, ed. 1991. Guide to yeast genetics and molecular biology. Academic Press, San Diego.

Harvey SL, Charlet A, Haas W, Gygi SP, Kellogg DR. 2005. Cdk1-dependent regulation of the mitotic inhibitor Wee1. Cell 122: 407-420.

Hauf S, Cole RW, LaTerra S, Zimmer C, Schnapp G, Walter R, Heckel A, van Meel J, Rieder CL, Peters JM. 2003. The small molecule Hesperadin reveals a role for Aurora B in correcting kinetochore-microtubule attachment and in maintaining the spindle assembly checkpoint. J Cell Biol 161: 281-294.

Hirano T. 2012. Condensins: universal organizers of chromosomes with diverse functions. Genes Dev 26: 1659-1678.

Holt LJ, Tuch BB, Villen J, Johnson AD, Gygi SP, Morgan DO. 2009. Global analysis of Cdk1 substrate phosphorylation sites provides insights into evolution. Science 325: 1682-1686.

Kao L, Wang YT, Chen YC, Tseng SF, Jhang JC, Chen YJ, Teng SC. 2014. Global analysis of cde14 dephosphorylation sites reveals essential regulatory role in mitosis and cytokinesis. Mol Cell Proteomics 13: 594-605.

Kimura K, Hirano M, Kobayashi R, Hirano T. 1998. Phosphorylation and activation of $13 \mathrm{~S}$ condensin by Cdc2 in vitro. Science 282: 487-490.

Kinoshita E, Kinoshita-Kikuta E, Takiyama K, Koike T. 2006. Phosphate-binding tag, a new tool to visualize phosphorylated proteins. Mol Cell Proteomics 5: 749-757.

Koivomagi M, Valk E, Venta R, Iofik A, Lepiku M, Morgan DO, Loog M. 2011. Dynamics of Cdk1 substrate specificity during the cell cycle. Mol Cell 42: 610-623.
Lavoie BD, Hogan E, Koshland D. 2004. In vivo requirements for rDNA chromosome condensation reveal two cell-cycle-regulated pathways for mitotic chromosome folding. Genes Dev 18: $76-87$.

Lenart P, Petronczki M, Steegmaier M, Di Fiore B, Lipp JJ, Hoffmann M, Rettig WJ, Kraut N, Peters JM. 2007. The small-molecule inhibitor BI 2536 reveals novel insights into mitotic roles of polo-like kinase 1. Curr Biol 17: 304-315.

Li Y, Cross FR, Chait BT. 2014. Method for identifying phosphorylated substrates of specific cyclin/cyclin-dependent kinase complexes. Proc Natl Acad Sci 111: 11323-11328.

Loog M, Morgan DO. 2005. Cyclin specificity in the phosphorylation of cyclin-dependent kinase substrates. Nature 434: 104-108.

Lyons NA, Morgan DO. 2011. Cdk1-dependent destruction of Ecol prevents cohesion establishment after S phase. Mol Cell 42: 378-389.

Maeshima K, Eltsov M. 2008. Packaging the genome: the structure of mitotic chromosomes. J Biochem 143: 145-153.

Marko JF. 2008. Micromechanical studies of mitotic chromosomes. Chromosome Res 16: 469-497.

Morgan DO. 2007. The cell cycle: principles of control. New Science Press, London.

Morishita J, Matsusaka T, Goshima G, Nakamura T, Tatebe H, Yanagida M. 2001. Bir1/Cut17 moving from chromosome to spindle upon the loss of cohesion is required for condensation, spindle elongation and repair. Genes Cells 6: 743-763.

Mortensen EM, Haas W, Gygi M, Gygi SP, Kellogg DR. 2005. Cdc28-dependent regulation of the Cdc5/Polo kinase. Curr Biol 15: 2033-2037.

Naumova N, Imakaev M, Fudenberg G, Zhan Y, Lajoie BR, Mirny LA, Dekker J. 2013. Organization of the mitotic chromosome. Science 342: 948-953.

Neurohr G, Naegeli A, Titos I, Theler D, Greber B, Diez J, Gabaldon T, Mendoza M, Barral Y. 2011. A midzone-based ruler adjusts chromosome compaction to anaphase spindle length. Science 332: 465-468.

Ono T, Fang Y, Spector DL, Hirano T. 2004. Spatial and temporal regulation of condensins I and II in mitotic chromosome assembly in human cells. Mol Biol Cell 15: 3296-3308.

Paulson JR. 2007. Inactivation of Cdk1/Cyclin B in metaphasearrested mouse FT210 cells induces exit from mitosis without chromosome segregation or cytokinesis and allows passage through another cell cycle. Chromosoma 116: 215225.

Pearlman SM, Serber Z, Ferrell JE Jr. 2011. A mechanism for the evolution of phosphorylation sites. Cell 147: 934-946.

Peplowska K, Wallek AU, Storchova Z. 2014. Sgol regulates both condensin and Ipl1/Aurora B to promote chromosome biorientation. PLoS Genet 10: e1004411.

Robbins JA, Cross FR. 2010. Requirements and reasons for effective inhibition of the anaphase promoting complex activator CDH1. Mol Biol Cell 21: 914-925.

Sanchez-Diaz A, Nkosi PJ, Murray S, Labib K. 2012. The mitotic exit network and Cdc14 phosphatase initiate cytokinesis by counteracting CDK phosphorylations and blocking polarised growth. EMBO I 31: 3620-3634.

Schott EJ, Hoyt MA. 1998. Dominant alleles of Saccharomyces cerevisiae CDC20 reveal its role in promoting anaphase. Genetics 148: 599-610.

Slaughter BD, Schwartz JW, Li R. 2007. Mapping dynamic protein interactions in MAP kinase signaling using live-cell fluorescence fluctuation spectroscopy and imaging. Proc Natl Acad Sci 104: 20320-20325. 
Stern B, Nurse P. 1996. A quantitative model for the cdc2 control of $\mathrm{S}$ phase and mitosis in fission yeast. Trends Genet 12: $345-350$.

St-Pierre J, Douziech M, Bazile F, Pascariu M, Bonneil E, Sauve V, Ratsima H, D'Amours D. 2009. Polo kinase regulates mitotic chromosome condensation by hyperactivation of condensin DNA supercoiling activity. Mol Cell 34: 416-426.

Strickfaden SC, Winters MJ, Ben-Ari G, Lamson RE, Tyers M, Pryciak PM. 2007. A mechanism for cell-cycle regulation of MAP kinase signaling in a yeast differentiation pathway. Cell 128: 519-531.

Sutani T, Yuasa T, Tomonaga T, Dohmae N, Takio K, Yanagida M. 1999. Fission yeast condensin complex: essential roles of non-SMC subunits for condensation and Cdc2 phosphorylation of Cut3/SMC4. Genes Dev 13: 2271-2283.

Tada K, Susumu H, Sakuno T, Watanabe Y. 2011. Condensin association with histone $\mathrm{H} 2 \mathrm{~A}$ shapes mitotic chromosomes. Nature 474: 477-483.

Takemoto A, Maeshima K, Ikehara T, Yamaguchi K, Murayama A, Imamura S, Imamoto N, Yokoyama S, Hirano T, Watanabe $\mathrm{Y}$, et al. 2009. The chromosomal association of condensin II is regulated by a noncatalytic function of PP2A. Nat Struct Mol Biol 16: 1302-1308.

van Heemst D, James F, Poggeler S, Berteaux-Lecellier V, Zickler D. 1999. Spo76p is a conserved chromosome morphogenesis protein that links the mitotic and meiotic programs. Cell 98: 261-271.

Vassilev LT, Tovar C, Chen S, Knezevic D, Zhao X, Sun H, Heimbrook DC, Chen L. 2006. Selective small-molecule inhibitor reveals critical mitotic functions of human CDK1. Proc Natl Acad Sci 103: 10660-10665.

Wilkins BJ, Rall NA, Ostwal Y, Kruitwagen T, Hiragami-Hamada K, Winkler M, Barral Y, Fischle W, Neumann H. 2014. A cascade of histone modifications induces chromatin condensation in mitosis. Science 343: 77-80.

Yeong FM, Hombauer H, Wendt KS, Hirota T, Mudrak I, Mechtler $\mathrm{K}$, Loregger T, Marchler-Bauer A, Tanaka K, Peters JM, et al. 2003. Identification of a subunit of a novel Kleisin- $\beta / S M C$ complex as a potential substrate of protein phosphatase $2 \mathrm{~A}$. Curr Biol 13: 2058-2064.

Yu HG, Koshland D. 2005. Chromosome morphogenesis: condensin-dependent cohesin removal during meiosis. Cell 123: 397-407. 


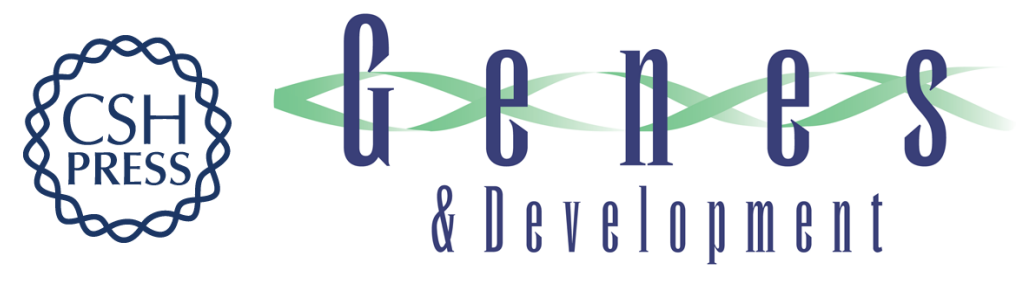

\section{A high-sensitivity phospho-switch triggered by Cdk1 governs chromosome morphogenesis during cell division}

Xavier Robellet, Yogitha Thattikota, Fang Wang, et al.

Genes Dev. 2015, 29:

Access the most recent version at doi:10.1101/gad.253294.114

\section{Supplemental http://genesdev.cshlp.org/content/suppl/2015/02/17/29.4.426.DC1 \\ Material}

References This article cites 53 articles, 24 of which can be accessed free at: http://genesdev.cshlp.org/content/29/4/426.full.html\#ref-list-1

Creative This article is distributed exclusively by Cold Spring Harbor Laboratory Press for the first Commons six months after the full-issue publication date (see

License http://genesdev.cshlp.org/site/misc/terms.xhtml). After six months, it is available under a Creative Commons License (Attribution-NonCommercial 4.0 International), as described at http://creativecommons.org/licenses/by-nc/4.0/.

Email Alerting Receive free email alerts when new articles cite this article - sign up in the box at the top Service right corner of the article or click here.

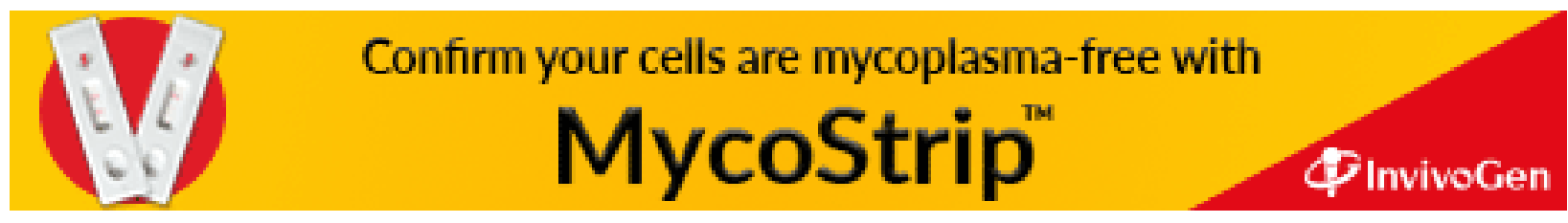

